IOSR Journal of Pharmacy

ISSN: 2250-3013, www.iosrphr.org

Volume 2 Issue 5 \|ा| Sep-Oct. 2012 | PP.11-14

\title{
A case of lupus pneumonitis mimicking as infective pneumonia
}

\author{
Dr Deependra Kumar Rai ${ }^{1}$, Dr Suneet K. Upadhyaya ${ }^{2}$, Dr Archana Sharma ${ }^{3}$, \\ Dr Krishna Kuldeep ${ }^{4}$
}

Assistant Professor, Department of Chest $\& \mathrm{~TB}^{1}$, Associate professor, Department of Psychiatry ${ }^{2}$, Assistant Professor, Department of Obstetrics And Gynecology ${ }^{3}$, Associate Professor, Department of Ophthalmology ${ }^{4}$, VCSG Govt. Medical science and research institute, Srinagar Garhwal, Uttarakhand.

\begin{abstract}
We report a case of Acute Lupus pneumonitis mimicking as Infective pneumonia .This patients admitted with short duration of symptom like dyspnea on exertion, cough with minimum expectoration and fever .Initially patients treated with antibiotic keeping the possibilities bacterial pneumonia but didn't respond and after further investigation patients diagnosed as acute Lupus pneumonitis. Patients significantly improved with course of corticosteroid. We should keep high degree of suspicion for lupus pneumonitis in young female with non resolving pneumonia .
\end{abstract}

Keywords—Acute Lupus pneumonitis, Systemic Lupus erythematosus

\section{INTRODUCTION}

Systemic Lupus erythematosus (SLE) is characterized by production of antibodies against various cellular antigens derived from nucleus, cytoplasm and cell membrane. Pulmonary manifestations of SLE can include a wide spectrum of diseases such as pleuritis, pneumonia, pulmonary embolism, pneumothorax and pulmonary hemorrhage. ${ }^{1-2}$ The pulmonary complications are thought to be the result of an immune complexmediated injury. Infection is most common form of pulmonary involvement in patients of SLE ${ }^{3}$. Acute lupus pneumonitis mimics an acute infectious pneumonia and incidence varies from $0.9 \%$ to $11.7 \%{ }^{2}$. The most common symptom of Acute lupus pneumonitis are dyspnea, dry cough, Fever and rarely Hemoptysis. ${ }^{4}$ A high index of suspicion should be maintained for the young female patient presenting with unexplained pulmonary infiltrates. The risk of lupus pneumonitis is very high in postpartum period. ${ }^{5}$ Blood and sputum cultures are necessary, and bronchoscopy or open-lung biopsy may be needed to exclude other conditions, such as pneumonia, alveolar hemorrhage, and other acute pulmonary processes that resemble lupus pneumonitis clinically and radio graphically ${ }^{\circ}$.

\section{CASE REPORT}

A 23 year old female admitted in January 2012 with shortness of breath, generalized body weakness and loss of appetite for last two month. There was history of multiple joint pain, Cough with minimum mucoid expectoration, more during night and low grade fever for last 2 weeks. There was no history of evening rise of temperature, significant weight loss, palpitation, orthopnea, hemoptysis, pedal edema. Bowel and bladder habits were within normal limit. Past medical history was unremarkable. She denied exposure to gases, fumes, toxic exposure.

At admission the patients had dyspnea with respiratory rate of 24 per minute, pulse 94 per minute, BP 108/ $66 \mathrm{~mm} \mathrm{Hg}$, Temperature 97.4 degree Fahrenheit with normal hydration. SpO2 was 94\% at room air. Chest examination revealed bilateral fine crackles. Cardiovascular examination revealed normal heart sound and no murmur. Abdominal finding were normal. There were no sign of lower extremity deep vein thrombosis. Chest $\mathrm{x}$-ray suggestive of bilateral patchy consolidation, predominant in lower zone.(figure 1) Chest CT scan showed well demarcated hypo dense area with linear fluid \& air filled structure seen in bilateral lung field. Image morphology may be secondary to pulmonary consolidation, no evidence of mediastinal lymphadenopathy.(figure 4) Routine Blood examination

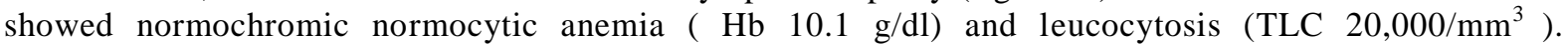
Differential count showed polymorph $94 \%$ and lymphocyte 5\%. Erythrocyte sedimentation rate $39 \mathrm{~mm}$,

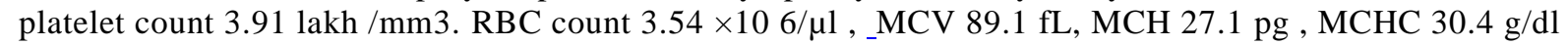
.Sputum for AFB by Zeel Neelson microscopy was negative. Sputum AFB culture by MGIT was negative. There was no pyogenic bacteria found in sputum culture, sputum for fungus culture shows growth of candida spp. She was negative for retroviral test. Her liver function tests and were normal 
except low serum protein values (Serum total protein $4.8 \mathrm{~g} / \mathrm{dl}$, serum albumin $1.6 \mathrm{~g} / \mathrm{dl}$, ) Other blood Investigation showed, serum TIBC $65.10 \mu \mathrm{g} / \mathrm{dl}$,_serum iron $16.00 \mu \mathrm{g} / \mathrm{dl}$, serum . Urine examination showed no sugar, trace of albumin, pus cell 2-4/hpf, and epithelial cells $1-2 / \mathrm{hpf}_{\text {. }}$
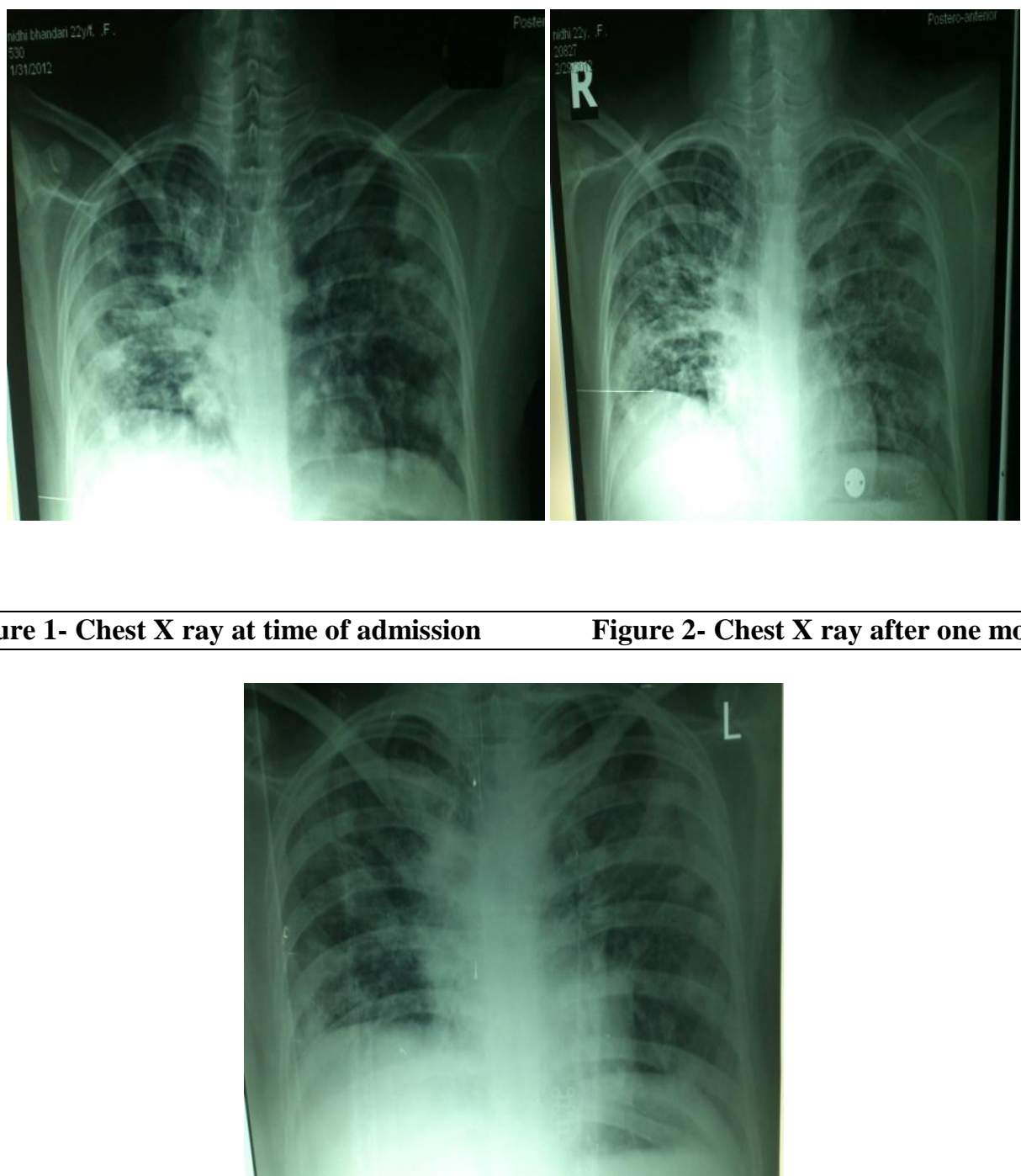

Fig 3-Chest $X$ ray after 6 months of treatment
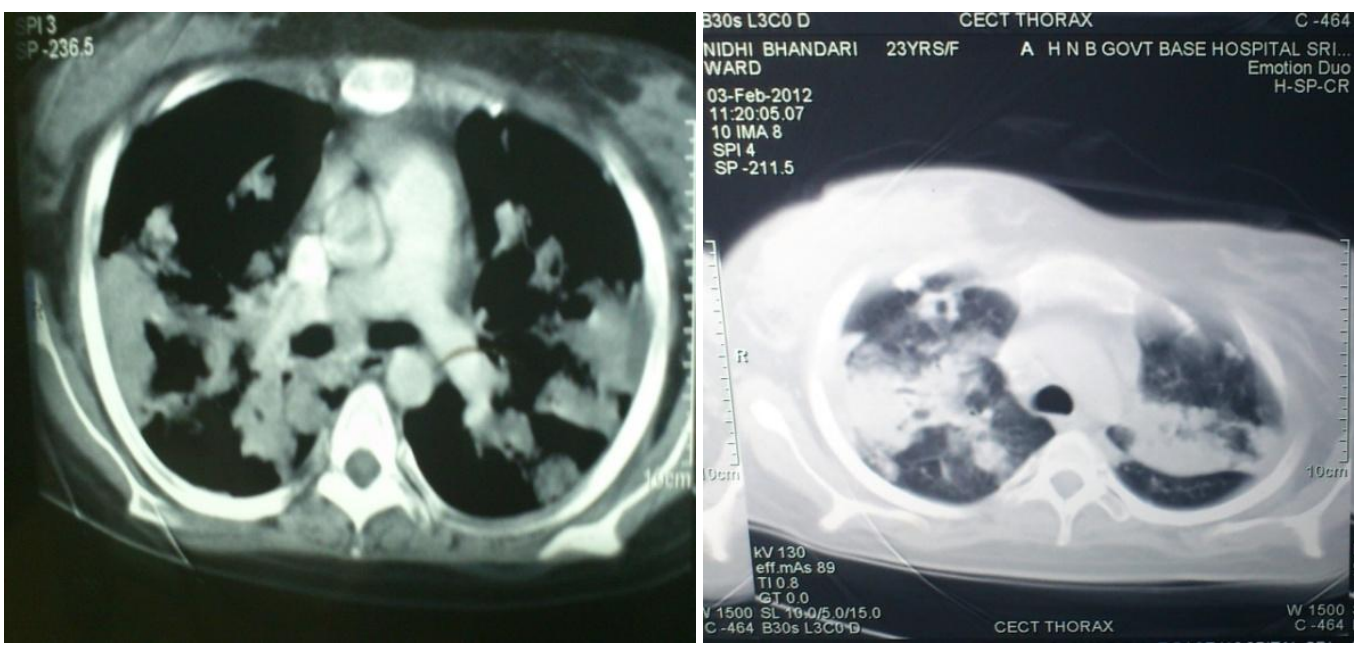

Fig -4 CT scan chest at time of admission 


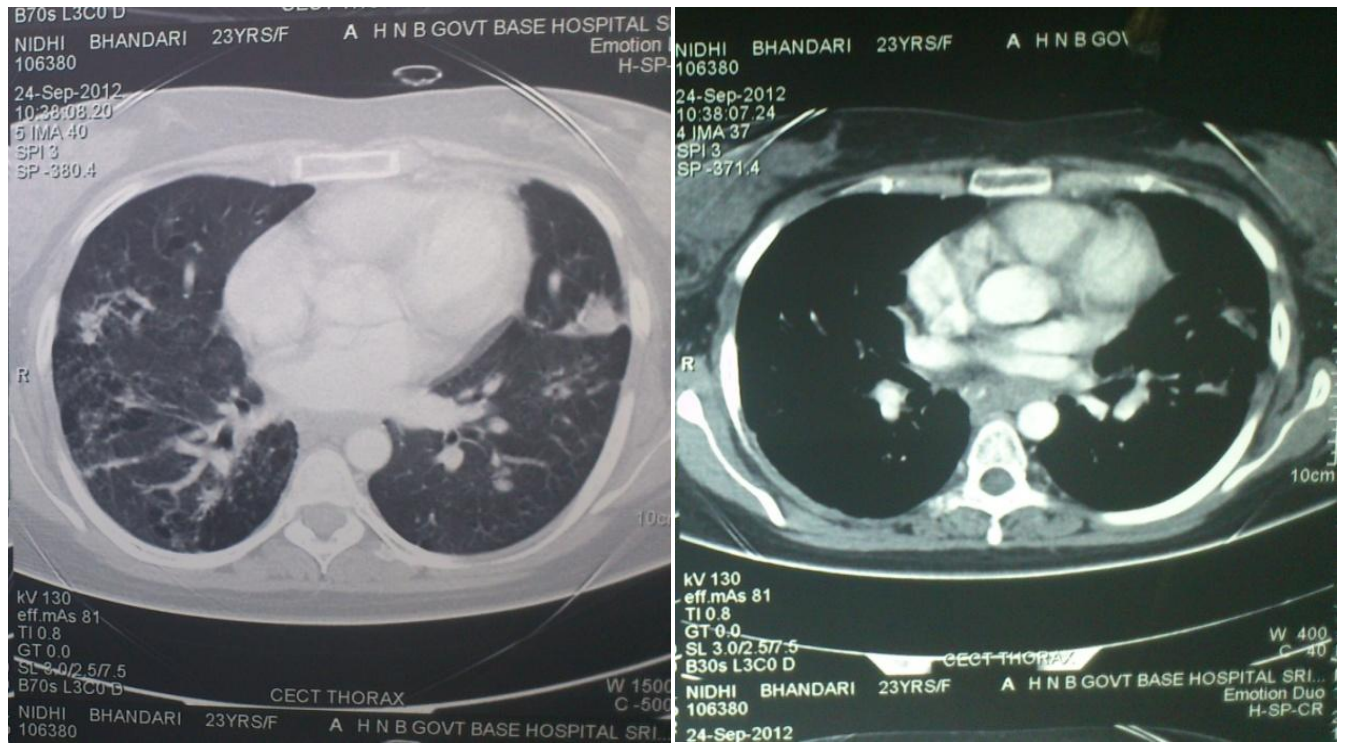

Fig 5- CT scan chest after 6 months of treatment

The provisional diagnosis of bacterial pneumonia was made and treatment started with intravenous Antibiotics ( ceftriaxone $1 \mathrm{gm}$ twice a day and Azithromycin 500mg once a day with supportive treatment. Even after 10 days of above regimen condition didn't improve much. On $\mathrm{D}_{5}$ ( days five) of admission her condition not much improved and she felt same degree of weakness, shortness of breath \& low appetite, When we repeat complete blood count it showed $\mathrm{Hb} 7.5 \mathrm{~g} / \mathrm{dl}$, TLC 11,900/mm3, differential count showed $80 \%$ polymorph, 15\% lymphocyte, and 5\% monocyte General blood picture showed normochromic normocytic picture along with microcytic, hypochromic at places with moderate anisonucleasis, punctuate basophilia-, WBC count approximately $11,900 / \mathrm{mm} 3$ differential count showed polymorph predominant with adequate platelets, no immature cell, hem parasite seen. At Day 10 of hospitalization patient received one unit of whole blood transfusion as no packed cells were available. Patient was discharged one day later on request of patients attendant and put on Tablet cefodoxime+ clavunic acid(200mg) and Tab azithromycin (500mg) for 10 days . Patient was reviewed two weeks later. There was no significant improvement either clinically \& radiological (figure 2). Then she was investigated for collagen vascular disease (CVD) associated lung diseases specially lupus pneumonitis. An antinuclear antibody (ANAs) was positive. Anti-dsDNA was $118 \mathrm{IU} / \mathrm{ml}$ (normal <60 $\mathrm{IU} / \mathrm{ml}$ ). Autoantibodies such as c- ANCA, Anti-GBM were negative. His clotting profiles and screening for lupus anticoagulants were negative. C reactive protein was $2.4 \mathrm{mg} / \mathrm{dl}$ (positive ). Flexible bronchoscopy (FBS) performed and $20 \mathrm{ml}$ bronchial aspirates was taken from Right lower lobe which was reddish in color, turbid, and without coagulum. Zeihl nelson staining for acid fast bacilli (AFB), AFB culture by mycobacterium growth indicator tube (MGIT), and PCR for Mycobacterium in bronchial aspirate were negative. The final diagnosis of Acute lupus pneumonitis was made and patient put on prednisolone 60mg once a day ,Iron supplement in form of syrup and tablet pantoprazole $40 \mathrm{mg}$ once a day._After two weeks of treatment she had partial improvement in dyspnea but low appetite and cough were persistent. The dose of $60 \mathrm{mg} / \mathrm{day}$ was given for two month and then taper over next six month to $10 \mathrm{mg} /$ day. In her next follow up visit one month later she reported significant improvement in all symptoms. At 6 months follow up, Chest x ray (figure 3) \& CT scan chest ( figure 5) showed almost $80 \%$ disappearance of radiological lesion, and she was asymptomatic at $10 \mathrm{mg}$ prednisolone/day.

\section{DISCUSSION}

Acute lupus pneumonitis is a clinical syndrome with an underlying histology of diffuse alveolar damage (DAD), bronchiolitis obliterans organizing pneumonia(BOOP), non specific interstitial pneumonia(NSIP), or a combination of these. Lupus pneumonitis was initial manifestation in SLE in half of twelve patients in one series, and most patients with lupus pneumonitis have established SLE at presentation. ${ }^{4}$ The mortality in Lupus pneumonitis may be as high as $50 \%{ }^{4}$. A high index of suspicion should be maintained for the young female patient presenting with unexplained pulmonary infiltrates. The number of investigation like sputum culture, blood culture and Fibreoptic bronchoscopy are required for conditions such as infective pneumonia, alveolar hemorage, and other condition that resembles to Lupus pneumonitis clinically and radiologically. ${ }^{7}$ This patients shown negative sputum and Blood culture. Bronchial aspirates culture didn't shows bacterial, mycobactrium, and fungal growth favoring for diagnosis of non infective pneumonitis. Our patient had cough with minimum expectoration, and dyspnea on exertion. There was no history of hemoptysis in 
our patients. The alveolar hemorrhage usually presents as hemoptysis, falling hematocrit, and pulmonary infiltrates. ${ }^{8} \quad$ Our patient had falling hematocrit, and bilateral diffuse infiltrates but did not have haemoptysis. Some patients of alveolar hemorrhage may not have hemoptysis ${ }^{9}$. Antinuclear antibodies (ANAs) are present in almost all cases of SLE ${ }^{10}$. Presence of ANAs and anti ds DNA in our patient was confirmatory for diagnosis of lupus pneumonitis. Presence of fever is usually suggestive of infective etiology, but fever may be present in 26 to 83 percentage cases of lupus pneumonitis. ${ }^{8}$ The other condition that come in differential diagnosis of acute lupus pneumonitis should be excluded such as Good Pasteur's syndrome and Wegner granulomatosis . In our case this condition was excluded on basis on negative c- ANCA ( anti neutrophilic cytoplasmic antibodies) and Anti GBM ( Glomerular basement membrane) autoantibodies. Fibreoptic bronchoscopy in our case showed reddish bronchial aspirates but not grossly bloody aspirates, and presence of hemosideren laden macrophages may shows presence of hemorrhage. ${ }^{12}$ This patients responded well with prednisolone similar to other studies ${ }^{8}$.

\section{CONCLUSION}

A high index of suspicion should be maintained for the young female patient presenting with unexplained pulmonary infiltrates. The early use of Fibreoptic bronchoscopy and bronchial aspirates examination helps in exclusion of infection so that patients can be put on corticosteroid as soon as possible .

\section{REFERENCES}

[1]. Beresford MW, Cleary AG, SillsJA, Couriel J, Davidson JE: Cardio-pulmonary involvement in juvenile systemic lupus ery-thematosus. Lupus2005, 14(2):152-8.

[2]. Ciftçi E, Yalçinkaya F, Ince E, Ekim M, Ileri M, Orgerin Z, Fitöz S, Güriz H, Aysev AD, Dogru U: Pulmonary involvement in childhood-onset systemic lupus erythematosus: a report of five cases. Rheumatology2004, 43(5):587-91.

[3]. Murin S, Wiedemann HP, Matthay RA. Pulmonary manifestations of systemic lupus erythema-tosus. Clin Chest Med 1998;19(4):641-65 [viii.].

[4] . Matthay RA, Schwarz MI, Petty TL, et al. Pulmonary manifestations of systemic lupus erythematosus: review of twelve cases of acute lupus pneumonitis. Medicine (Baltimore) 1975;54(5):397-409

[5]. Trager J, Ward MM. Mortality and causes of death in systemic lupus erythematosus. Curr OpinRheumatol 2001;13(5):345-51

[6]. Haupt HM, Moore GW, Hutchins GM. The lung in systemic lupus erythematosus. Analysis ofthe pathologic changes in 120 patients. Am J Med 1981;71(5):791-8

[7]. Haupt HM, Moore GW, Hutchins GM. The lung in systemic lupus erythematosus. Analysis of the pathologic changes in 120 patients. Am J Med 1981;71(5):791-8

[8]. Zamora MR, Warner ML, Tuder R, et al. Diffuse alveolar hemorrhage and systemic lupus erythematosus. Clinical presentation, histology, survival, and outcome. Medicine (Baltimore) 1997;76(3):192-202

[9]. Santos-Ocampo AS, Mandell BF, Fessler BJ. Alveolar hemorrhage in systemic lupus erythema-tosus: presentation and management. Chest 2000;118(4):1083-90.

[10]. Roark JH, Kuntz CL, Nguyen KA, et al. Breakdown of B cell tolerance in a mouse model of systemic lupus erythematosus. J Exp Med 1995;181(3):1157-67

[11]. Abud-Mendoza C, Diaz-Jouanen E, Alarcon-Segovia D. Fatal pulmonary hemorrhage in systemic lupus erythematosus. Occurrence without hemoptysis. J Rheumatol 1985;12(3): 558-61

[12]. Leatherman JW, Davies SF, Hoidal JR. Alveolar hemorrhage syndromes: diffuse microvascular lung hemorrhage in immune and idiopathic disorders.Medicine (Baltimore) 1984;63(6):343-61. 